\title{
PERSPECTIVA ACTUAL DE LA PROMOCIÓN DE ACTIVIDAD FÍSICA PARA LA SALUD EN NIÑOS Y ADOLESCENTES EN EL CONTEXTO ESCOLAR. REVISIÓN
}

\section{CURRENT PERSPECTIVE OF THE PROMOTION OF PHYSICAL ACTIVITY IN CHILDREN AND ADOLESCENTS IN SCHOOL CONTEXT. REVIEW}

\author{
Jairo Alejandro Fernández Ortega ${ }^{1}$ \\ Luz Amelia Hoyos Cuartas ${ }^{2}$
}

\section{Resumen}

La escuela es considerada el escenario ideal para intervenir sobre las condiciones de salud de niños y jóvenes; por ello ha sido el centro de múltiples programas diseñados a partir de perspectivas teóricas diversas, que van desde los enfoques biologistas hasta los de medicina social, con la esperanza de impactar en las condiciones de calidad de vida. El propósito del estudio fue analizar e identificar los diferentes componentes utilizados (modelo, estrategias, agentes involucrados y duración) en el diseño de programas de promoción de actividad física (AF) para la salud en el contexto escolar, por lo cual se analizaron diferentes estudios que desarrollaron programas de AF en este aspecto. La evidencia observada indica una gran diversidad en el diseño y componentes de las intervenciones. A pesar de los resultados positivos obtenidos, se identificó heterogeneidad: en la metodología, la población, los actores involucrados y en los indicadores de eficacia, lo cual no permite realizar comparaciones entre los diferentes estudios. Es necesario que futuras intervenciones involucren indicadores individuales y colectivos, que su acción sea de orden longitudinal implicando a todos los actores que inciden en diferentes niveles de intervención.

Palabras claves: programas de promoción de la salud, actividad física, niños, adolescentes.

\section{Abstract}

The school is considered as an ideal to speak on the health conditions of children and young people, this has been the focus of many programs designed from different theoretical perspectives, ranging from biologists approaches to approaches of social medicine with the scenario hope to impact the quality of life conditions. The purpose of this study is to analyze identify and understand the guidelines axes, strategies, actors involved and duration, which have guided the PA promotion programs in children and adolescents in the school context. Method: the different studies which developed PA programs directed towards health in the school context were analyzed. Results: The observed evidence suggests a great heterogeneity in the design and components of interventions. Conclusions: Despite the positive results, heterogeneity was identified: the methodology, population, stakeholders and performance indicators, which do not allow comparisons between different studies. It is necessary that future interventions indicators involve individual and collective, that their action is longitudinal order and involve all stakeholders who have different levels of impact on the consolidation of these interventions.

Keywords: programs of health promotion, PA, children, adolescents.

$1 \mathrm{PhD}$ en Ciencias de la Actividad Física y el Deporte. Magíster en Fisiología del Ejercicio. Docente de Planta Universidad Pedagógica Nacional. Centro de Investigaciones en Actividad Física y Deporte (CIDER). Correo electrónico: jairofdz@pedagogica.edu.co

$2 \mathrm{PhD}$ en Ciencias de la Actividad Física y el Deporte. Docente de Planta Universidad Pedagógica Nacional, Facultad de Educación Física. Correo electrónico: luzahoyos@yahoo.com 
Fecha de recepción: 15 de mayo de 2015

Fecha de aprobación: 1 de octubre de 2015

Para citar este artículo:

Fernández, J.A. y Hoyos, L.A. (2015). Perspectiva actual de la promoción de actividad física para la salud en niños y adolescentes en el contexto escolar. Revisión. Lúdica Pedagógica, (22), 85-99.

\section{INTRODUCCIÓN}

Los estudios epidemiológicos ponen en evidencia la problemática de la inactividad física, como una de las situaciones más complejas para la salud pública en el mundo debido a su estrecha relación con la aparición de enfermedades crónicas degenerativas, que han incrementado la carga por morbimortalidad en el mundo y que afecta a hombres y mujeres de todas las edades, estratos socioeconómicos y de diversas culturas. El síndrome de muerte por sedentarismo sugiere que este estilo de vida incide en el aumento de las tasas de mortalidad, y tiene su génesis durante la niñez y adolescencia (Fernández y Hoyos, 2007).

Las enfermedades cardiovasculares tienen sus inicios en la infancia (Harrell et al., 1998), es decir los factores de riesgo de enfermedades coronarias e infarto se presentan con gran prevalencia en los niños y adolescentes, los cuales tienden a dejar vestigios o permanecer durante la edad adulta. Investigaciones longitudinales han demostrado que el sedentarismo pediátrico está asociado con un mayor predominio de hipertensión, dislipidemia, resistencia a la insulina y el desarrollo de la obesidad infantil, como lo afirma el Centers for Disease Control and Prevention (CDC, 2000) y autores como Warren, Lightowler, Bradshawy Perwaiz (2003).

Por otra parte, el sobrepeso durante la niñez y adolescencia están fuertemente asociados con sobrepeso en la adultez (McKenzie, Feldman, Woods y Romero, 1995). Un estudio realizado en Estados Unidos por el Centro Nacional de Salud y Nutrición (NHANES) demostró que el 20,6 \% de los niños de 2 a 5 años, el 30,3 \% de los de 6 a 11 años, y el 30,4\% de adolescentes y adultos jóvenes de 12 a 19 años, se encuentran en sobrepeso o en riesgo de adquirirlo, siendo más propensos los niños afrodescendientes e hispanos. Un estudio realizado en Bogotá con 50.000 escolares detectó que el 7,9\% de estos niños se encuentra en riesgo de sobrepeso y el 8,8 \% tiene sobrepeso (Fernández y Ruiz, 2012).
La actividad física (AF) durante la niñez y adolescencia ha sido el centro de atención de diversos estudios pediátricos a partir de la década pasada. La salud física y psicológica del niño está íntimamente ligada a la realización de AF de manera regular (Skyboy y Ryan, 2002). Se ha establecido igualmente la importancia de la AF en el crecimiento y desarrollo armónico, en la disminución de exposición a agentes no saludables, en el desempeño académico (Mummery, Spencer y Hudec, 2000) y como mecanismo para instaurar hábitos de vida saludables, debido a que patrones de AF adquiridos en edades tempranas, van a repercutir en los hábitos y estilos de vida en la edad adulta disminuyendo de esta forma los riesgos de aparición de enfermedades crónicas cardiovasculares y metabólicas (McKenzie, et al., 1995; McKenzie et al., 1997; Wu y Jwo, 2005; Stewart, Dennison, Kohl y Doyle, 2004; McBride, Midford, y Cameron, 1999; Higgings, Gaul, Gibbons y Gyn, 2003; Fowler-Brown y Kahwati, 2004). Por ello, la promoción de la AF en los niños emerge como una de las estrategias de prevención más sólidas para contrarrestar los efectos nocivos de la inactividad física (Fowler-Brown y Kahwati, 2004) convirtiéndose en componente esencial dentro de los nuevos conceptos de calidad de vida y como un elemento primordial del desarrollo humano propuestos por la Organización Mundial de la Salud.

Sin embargo, a pesar de esta evidencia, la disminución en los niveles de AF comienza al final de la escuela elemental y continúa durante el bachillerato hasta la adultez; los datos estadísticos muestran que solo un 33 \% de la población escolar realiza ejercicio físico vigoroso tres veces por semana (Felton, Saunders, Wardy Dishman, 2005). Los estudios desarrollados por el CDC sobre la cantidad de adolescentes que realizan AF, reportan que el porcentaje pasó del 42 \% en 1991 al $25 \%$ en 1995. Esta situación es más preocupante en mujeres que en hombres; así lo demuestra una investigación con 201 mujeres jóvenes con o en riesgo de sobrepeso (McBride y Midford, 1999). Otro estudio realizado con 576 estudiantes de quinto a octavo grado 
de bachillerato; encontró que menos del $25 \%$ de estos niños realizan AF en forma cotidiana, y que los niveles de AF disminuyen constantemente a través de la adolescencia, más en niñas que en niños (Agron, Takada y Purcell, 2002).

Las causas del descenso en los niveles de AF en los niños y adolescentes son múltiples, debido a que la inactividad física se presenta por causas multifactoriales. Mendoza, Ságrera y Batista (1994) plantean que existen cuatro tipos de factores que determinan el estilo de vida de una persona; las características individuales, genéticas o adquiridas; y las características del entorno microsocial del individuo (vivienda, familia, escuela); existen también otros factores macrosociales que influyen directamente sobre los anteriores: el sistema social, la cultura predominante, los medios de comunicación y el medio físico geográfico, los factores socioeconómicos y el empleo de los padres (Hill y Quam, 2003).

\section{PROGRAMAS DE PROMOCIÓN DE AF EN ESCOLARES}

Los expertos en salud pública han identificado la escuela como el escenario ideal para la implementación de programas de AF, que conduzcan a la construcción de hábitos de vida saludables en niños y jóvenes, como se observa en la revisión sistemática realizada por Dobbins, Husson, DeCorby y LaRocca (2013). Estos programas incorporan una gran variedad de estrategias para incrementar la AF de los escolares como; evaluación regular de cualidades físicas, desarrollo de centros de AF escolar, enseñanza de conceptos de salud, motivando a los estudiantes a ser activos dentro y fuera de la clase de educación física y utilizando recursos tecnológicos para evaluar la condición física y los niveles de actividad (Hill y Quam, 2003).

Desde la década de 1990, en Estados Unidos se han llevado a cabo diversos proyectos para incrementar la educación física en los colegios. En 1993 se publicó el documento América 2000, en el cual se presentaban los objetivos de educación a nivel nacional y se acentuaba la importancia que tiene la EF para la promoción de la condición física de los jóvenes. En 1995 la Asociación Nacional de Actividad Física y Deporte (NASPE) desarrolló los estándares nacionales de EF (Corbin y Pangrazi 2004) y trazó los lineamientos para un programa de promoción "físicamente activos, estilos de vida para jóvenes".
Como fruto de estos esfuerzos surgió el programa Sports, Play, and Active Recreation for Kids (Spark) desarrollado por la Universidad de San Diego, con apoyo del Instituto Nacional de Salud (Sallis et al., 1997; Levin y Martín, 2002; Dowda et al., 2005). La iniciativa se desarrolló en torno a tres componentes principales: currículo activo de EF, formación del personal docente y apoyo en el lugar. El currículo activo de EF tiene como objetivos, fomentar que las clases de EF estén encaminadas hacia la salud, promover altos niveles de AF durante las clases, fomentar la realización de AF durante el tiempo libre, enseñar habilidades de movimiento y diseñar actividades agradables. El programa de formación de los profesores de EF plantea tres objetivos básicos: ampliar con los docentes, el nivel de comprensión de la relación, "EF y salud"; ayudarles a entender las unidades curriculares y actividades de SPARK; desarrollar habilidades para el manejo e implementación efectiva del programa y asistirlos en el diseño de estrategias para sobrepasar las posibles barreras que puedan surgir para la ejecución.

Sallis et al. (1997) realizan un estudio en siete escuelas de primaria en el estado de California; allí observan la incidencia que tiene el nivel de formación del docente a cargo del programa SPARK. Participaron 955 estudiantes (53\% hombres), con una edad promedio de 9,6 años que se distribuyeron en tres grupos: 264 en el grupo dirigido por un especialista, 331 en el grupo dirigido por un profesor capacitado para la implementación del programa y 360 en el grupo control que realizaba sus clases de EF tradicionales. Los resultados del estudio señalan diferencias significativas $p>0,005$ entre los tres grupos. Los estudiantes del grupo control presentaron una baja participación en actividades físicas vigorosas y moderadas, tanto en frecuencia como en tiempo, lo cual condujo a un menor gasto energético $(3,3 \mathrm{kcal} / \mathrm{kg} / \mathrm{wk})$. Los estudiantes del grupo dirigido por el especialista realizaron actividades físicas vigorosas y moderadas de mayor duración y frecuencia, presentando un gasto energético de 7,2 kcal/kg/wk, seguido del grupo del profesor entrenado con 5,8 kcal/kg/wk. Sin embargo, el estudio reporta que no hubo un incremento en la $\mathrm{AF}$ fuera de la escuela.

El programa SPARK también fue implementado en diez colegios rurales de la India, donde existen grupos de minoría étnica y los adultos presentan alta prevalencia de sobrepeso e inactividad física. El estudio no reporta resultados frente a los niveles de AF, sin embargo, resalta 
la importancia que tienen las intervenciones tempranas de AF en el mejoramiento significativo de la salud (Reinhardt y Brevard, 2002).

Dowda et al. (2005) evalúan la implementación del programa SPARK en 111 escuelas primarias de siete estados de Estados Unidos y encontraron: que el $81 \%$ de los profesores encuestados afirmó estar aplicando actualmente el programa SPARK en sus escuelas; el apoyo de los rectores de los centros educativos para la implementación del programa; y la realización de tres clases de EF por semana. El 90 \% de los profesores que utilizaban el programa indicaron: facilidad en el acceso al libro SPARK y en la adaptación de las clases de EF al programa. Utilización de los conceptos, métodos y planes del SPARK en por los menos el $50 \%$ de sus clases, y el 80 \% reportó haber implementado el programa por un periodo superior a dos años.

Dependiendo del tipo de intervenciones que se realicen en la escuela, se puede tener incidencia en el comportamiento de los jóvenes, aun después de terminada su aplicación. Dale y Corbin (2000) evaluaron el impacto en los hábitos de AF a largo plazo, en un grupo de niños que participó el programa Project Active Teens (PAT) y un grupo que realizó clases tradicionales de Educación Física (EFT), cuando estaban cursando noveno grado. Para ello se aplicó la encuesta Youth Risk Behavior Survey (YRBS, Encuesta de riesgo comportamental en jóvenes) a este grupo de jóvenes cuando estaba en la universidad.

El proyecto Active Teens fue desarrollado por investigadores de la Universidad de Arizona, con el objetivo de incrementar la AF en niños y jóvenes. El programa estaba compuesto de tres grandes tipos de actividades: actividades teóricas y talleres en la clase de EF, donde se desarrollan conceptos, sobre AF y salud que tienen como objetivo promover una actitud positiva hacia la práctica de AF y a adoptar una forma de vida físicamente activa. Estas actividades se desarrollaban una vez a la semana en el salón de clase (actividades de coordinación), las cuales se realizaban una vez a la semana en el gimnasio y actividades deportivas tres días a la semana. El estudio indicó que un mayor número de estudiantes del grupo PAT mantuvo una AF moderada, frente a los que integraron el grupo EFT. El $65 \%$ de los estudiantes del grupo PAT realizaban actividades de alta intensidad, contra un 29 \% del grupo EFT. Por otra parte, en las mujeres se observó un $10 \%$ de sedentarismo en el PAT y $22 \%$ en el EFT.
Beets y Pitetti (2002) determinaron la contribución de la EF y del deporte escolar en la condición física de estudiantes de secundaria. Compararon la condición cardiovascular (CVF; VO2, pico), fuerza, resistencia de los miembros superiores, flexibilidad e índice de masa corporal, entre los estudiantes que participaron en el programa School-Sponsored Sport Activites y los que realizaban clases tradicionales de EF. El programa constaba de dos componentes; un currículo de EF centrado en el uso activo del tiempo libre en actividades deportivas de equipo, con énfasis en el fortalecimiento físico, mental, emocional y social. Las actividades se realizaron tres veces por semana con una duración de dos horas por sesión, durante el año escolar. Participaron 187 estudiantes, 120 hombres y 67 mujeres, con edades entre 14 y 19 años. Los principales hallazgos mostraron que tanto los hombres como las mujeres presentaron mejores resultados en: el VO2 pico con valores de $50,73 \mathrm{ml} /$ $\mathrm{min} / \mathrm{kg}$, en hombres, y $42,4 \mathrm{ml} / \mathrm{min} / \mathrm{kg}$ en mujeres y en la fuerza de miembros superiores 49 repeticiones en hombres y 22 en mujeres, frente a los que participaban únicamente en la clase de EF. En las variables de flexibilidad e índice de masa corporal no se hallaron diferencias significativas entre los dos grupos en los dos géneros.

El programa Calidad de EF Diaria (QDPE) desarrollado en 1986 por la Asociación Canadiense de Salud, Educación Física, Recreación y Danza (CAHPERD), referenciado por Chad, Humbert y Jackson (1999) incluyen en el currículo de EF temas de AF diaria, nutrición, programas intramurales y aspectos comportamentales relacionados con la condición física de los estudiantes de escuelas públicas rurales y urbanas de 11 provincias de Canadá. El programa presenta cuatro componentes: a) conciencia, b) implementación, c) reconocimiento d) investigación y evaluación. Los resultados indican que el programa generó gran interés en los estudiantes aunque con poca participación, debido a la falta de docentes especializados, la insuficiente variedad de actividades propuestas y las pocas horas de clase de EF a la semana. Adicionalmente, uno de los grandes problemas para su implementación, fue la falta de conocimiento del programa por parte de los profesores de EF en las escuelas.

En Irán, se implementó el proyecto Corazón Saludable, del Golfo Pérsico, basado en el programa Heart Power. El propósito del estudio era determinar si una intervención en el contexto escolar basada en adquisición de conceptos sobre un corazón saludable, mejora los niveles de AF 
y los conocimientos sobre la salud del corazón. Participaron 1200 estudiantes (600 en el grupo control y 600 en el de intervención); 49,1 \% niños y 50,9 \% niñas, de tercer y cuarto grado. Los dos grupos realizaron un test sobre conocimiento de salud del corazón. El grupo de intervención recibió dos horas semanales de clase sobre función cardiaca, nutrición, AF y vivir sin cigarrillo, durante ocho semanas. Los resultados indican que el 81,4 \% de los estudiantes del grupo de intervención aprobó el test al final del estudio, contra 56,4 \% de los estudiantes del grupo control, se observó un incremento en el conocimiento acerca de la salud para el corazón y en los niveles de AF (Nabipour et al., 2002).

En Inglaterra se realizó el Programa de Condición Física para Jóvenes "Ideal a Real", el cual se implementó como parte del currículo de las clases de EF. El programa tuvo como propósito promover en los niños conocimientos sobre: salud y condición física, actitudes saludables, incrementar los niveles de AF y adopción de estilos de vida saludables. El proyecto consta de una fase de diseño, y otra que de implementación del programa a partir unidades temáticas y módulos. Cada unidad está constituida por 15 módulos para desarrollar en la clase y en el hogar y aborda conceptos de salud y fitness, de acuerdo con el grado de escolaridad, por ejemplo: primero-segundo: conocimiento corporal, tercerocuarto: alimentación y fitness, quinto-sexto: estrategias de movilidad y salud, séptimo-octavo: campañas para prevenir el uso de tabaco y drogas. Los profesores de EF enseñaban de 10 a 20 minutos semanales temas relacionados con fitness y salud. Los resultados del estudio muestran que los estudiantes sí profundizaron sobre conceptos de salud y condición física e iniciaron cambios alimenticios e incrementaron los niveles de AF (Hutchinson, Freedson, Ward y Rippe, 1990).

Levin, Martin, McKenzie y DeLouise (2002) desarrollaron un estudio denominado dinamovimiento. Se trata de un video que tiene como objetivo promover: la AF, la adquisición de conocimientos de los beneficios de la $\mathrm{AF}$, la autoeficacia, la actitud positiva frente a la AF y los comportamientos saludables en los jóvenes. El estudio evaluó el impacto de observar este video durante 15 minutos tres veces a la semana sobre los conocimientos de salud cardiovascular, autoeficacia y la actitud frente a la AF. Participaron 208 estudiantes de diferentes niveles de una escuela pública de Maryland. Se realizaron pre- y postest y se designaron grupos control e intervención por curso. Se aplicó un cuestionario de nueve preguntas, de las cuales siete eran sobre anatomía, función cardiovascular y beneficios de la AF. Además, durante la intervención se evaluaron cualitativamente la motivación y los aprendizajes del grupo intervención. Los resultados muestran un mayor conocimiento del grupo intervención respecto a actividad física, mayor autoeficacia, mejor actitud positiva frente a la AF y aumento en la percepción de alegría, al igual que mayor interés y habilidad motora a partir de su participación en las danzas que indicaba el video.

El Programa Movimiento Temprano kinderGym, diseñado por la Universidad del Norte de Iowa, se basó en tres fundamentos: enseñanza, refuerzo escolar y experiencias de movimiento, para promover cambios comportamentales, relacionados con AF. En primera instancia se observa el desarrollo de los patrones motores de los niños y se orienta a los padres sobre las características del desarrollo motor y se les recomiendan actividades perceptivo-motrices y rítmicas, con tres sesiones por semana. Se conformaban grupos según la edad y al nivel de desarrollo. El estudio no presenta resultados de la intervención (Marston, 2002).

Igualmente se identificaron programas, que promueven formas de movilización alternativas para los escolares. Desde esta perspectiva surge en el Condado de Marilyn, California, el Programa de Rutas Seguras hacia la Escuela, el cual promueve el desplazamiento de los estudiantes al colegio caminando, o usando bicicleta, patines, tabla etc., con el propósito de incorporar la AF dentro de la rutina diaria de los niños. El programa identifica las diferentes vías de acceso hacia la escuela y, con la participación diferentes estamentos y miembros de la comunidad (padres de familia, docentes de EF y directivos del colegio), crea un sistema de rutas seguras. En el interior de la escuela implementa competencia de kilómetros entre los estudiantes, conceptos sobre estilos de vida saludables, y genera un proceso de difusión a través de folletos informativos. En el segundo año de su implementación, participaron 4665 estudiantes de 15 escuelas lo que condujo a un incremento del $64 \%$ en el número de niños caminando, $114 \%$ en el número de estudiantes montando bicicleta, y una disminución del $39 \%$ en la llegada a la escuela en vehículo particular (Staunton, Hubsmith, y Kallins, 2003). Este mismo tipo de estudios con intervención en los medios de transporte hacia la escuela fue implementado posteriormente por Grow et al. (2008) 
Harrell et al. (1998) implementaron un programa de AF en niños con múltiples factores de riesgo cardiovasculares. El objetivo era determinar los efectos que tiene implementar en las clases de EF, sesiones sobre riesgos de ECV, baja capacidad aeróbica y obesidad, en la salud. El programa se desarrolló durante ocho semanas, con tres sesiones por semana. Los resultados del estudio indican que se generaron efectos positivos sobre la salud de la población escolar intervenida, presentando disminución en los niveles de colesterol, en los valores de los pliegues cutáneos y en el porcentaje de grasa total y se incrementaron los conocimientos sobre salud.

En otro estudio, Harrell, McMurray, Gansky y Bangdiwala (1999) compararon los efectos de dos intervenciones dirigidas a niños de primaria, para mejorar la salud cardiovascular. La primera intervención está soportada en dos componentes: a) de conocimiento/actitud acerca de cómo seleccionar alimentos saludables para el corazón, los peligros de fumar, como combatir la presión para fumar, y la importancia de realizar AF regular; b) de realización de AF aeróbicas divertidas, no competitivas, que involucraran los principales grupos musculares, tres veces por semana. La segunda intervención estaba basada en el riesgo de ECV, y estaba dirigida exclusivamente a aquellos estudiantes que presentaban uno o más de factores de riesgo de ECV. Se realizaron tres grupos de clases: a) de nutrición, dictadas por enfermeras(os), para los estudiantes con obesidad o niveles altos de colesterol ( $n=393$ ); b) de AF, dictadas por educadores físicos, para los estudiantes en riesgo, por baja capacidad aeróbica ( $n=213)$; y clases de no comenzar a fumar, para los estudiantes en riesgo aumentado de fumar $(n=455)$. Los estudiantes podían participar de una o más temáticas si tenían múltiples factores de riesgo de base. Las clases se dictaron durante ocho semanas en grupos de cinco a ocho estudiantes durante el horario normal. Se observaron efectos significativos en el conocimiento del sistema cardiovascular, puntajes de AF autorreportada, los cuales, para las escuelas de intervención, se dieron en mayor proporción que en las escuelas control.

En la ciudad de Culver, California, se implementó en las escuelas de bachillerato el programa Teen Activity Project (TAP, Proyecto de actividad para adolescentes); una serie de talleres dirigidos a madres y niñas de las escuelas participantes, con información acerca de la importancia de la AF, y alimentación saludable. Se deter- minaron los factores principales para la participación en la AF y se desarrolló un programa que constaba de clases de EF y nutrición. Las niñas participantes mostraron un aumento en el conocimiento acerca de la condición física y las opciones saludables de alimentación, aunque los cambios reales en estas opciones no fueron significativos de acuerdo con lo evidenciado en la valoración dietaria pre- y postest (Leslie, Yancy, McCarthy y Albert, 1999). El programa Phyisical Activity and Teenage Health (РATH, Actividad física y salud de las adolescentes), de las escuelas de bachillerato en Nueva York, tenía como objetivo el cambio comportamental de las adolescentes. Estaba basado en programas de ejercicio vigoroso integrado con educación en nutrición y salud para promoción del cambio comportamental. Las clases eran de 30 minutos, 5 días/semana, durante 12 semanas. Se iniciaba con 5-10 minutos de información y discusión sobre la anatomía y fisiología del corazón, factores de riesgo cardiovasculares, procesos de enfermedades cardiacas, manejo adecuado de estrés, ejercicio y nutrición; cómo evitar el cigarrillo, técnicas para dejar de fumar, y estrategias para modificar los comportamientos de alto riesgo para la salud. Posteriormente, 20-25 minutos de AF vigorosa, con ejercicios para mejorar la fuerza y resistencia muscular, o ejercicio aeróbico para mejorar el estado cardiovascular. Los resultados del estudio indican diferencias significativas en el porcentaje de grasa corporal entre el grupo PATH y el grupo control $(29,7$ vs. $31,1, p<0,05)$. Igualmente se observó una autopercepción de salud un poco más alta en el grupo PATH (6,2 vs. 5,8, $p<0,05)$, al igual que diferencias importantes en cuanto al conocimiento de la salud cardiaca (Bayne et al., 2004).

El programa Play implementado en 1996 por el departamento de salud en escuelas de Arizona, tenía como objetivo enseñar a los niños de cuarto a sexto grado hábitos de estilos de vida saludables y estimularlos a acumular de 30 a 60 minutos diarios de AF moderada a vigorosa autodirigida, durante 12 semanas. Dos grupos participaron en el programa: el grupo Play-EF, y solo EF. El programa se implementó en tres fases:

- Fase 1. Promoción del comportamiento de juego. Durante una semana los profesores discutían con los alumnos la importancia de la Af y los procedimientos de Play, y juntos participaban en actividades de 15 minutos cada día escolar. 
- Fase 2. Introducción de actividades dirigidas por los maestros. Durante tres semanas profesores y estudiantes continuaban con los 15 minutos de $A F$, pero en esta fase los profesores implementaban juegos y actividades.

- Fase 3. Fomentar la actividad autodirigida. Durante ocho semanas se estimuló a los estudiantes para completar 30 minutos o más de AF independiente (sin la dirección o presencia del profesor), preferiblemente en actividades extraescolares. Los resultados del estudio indican que la intervención fue efectiva y se incrementaron los niveles de AF en los estudiantes, sobre todo en las niñas (Pangrazi, Beighle, Beige y Vack, 2003).

\section{MODELOS TEÓRICOS UTILIZADOS DENTRO DEL CONTEXTO ESCOLAR PARA LA PROMOCIÓN DE LA AF}

Para lograr el cambio de comportamiento y construcción de hábitos, se han diseñado una serie de modelos ${ }^{3}$ que intentan explicar comprender y definir los parámetros y factores que determinan estos comportamientos. Dichos modelos aportan las bases metodológicas y teóricas a partir de las cuales se diseñan los programas de intervención para la promoción de la AF y están encaminados a lograr cambios en el comportamiento de los niños y adolescentes a partir de los factores modificables. Cada uno de ellos con niveles de intervención, campos, agentes y estrategias diferentes (Fernández y Rocha, 2008). Varios estudios, en el campo de la promoción de la AF para la salud en el contexto escolar han utilizado estos modelos y la evidencia de sus resultados muestran su efectividad. Sin embargo, existe gran controversia sobre cuál de todos estos modelos y teorías es el más pertinente en el contexto escolar.

En el Reino Unido se llevó a cabo un programa en la escuela primaria diseñado a partir de la teoría del aprendizaje social de Bandura. El programa se fundamenta en: resaltar el valor del comportamiento

3 Teorías clásicas de aprendizaje, modelo de creencias en salud, modelo transteórico, teoría social cognitiva, teoría del comportamiento planificado, modelo de soporte social, modelo ecológico, la teoría social ecológica. deseado incluyendo beneficios a corto plazo, brindar incentivos para reforzar los mensajes (elogios verbales y pequeños premios), desarrollar habilidades prácticas y, por tanto, autoconfianza en el comportamiento deseado; trabajar con los padres, para superar las barreras que se oponen a comportamientos saludables. Los estudiantes se organizaron en cuatro grupos: a) de nutrición (Be Smart), en el que se trataron temas de comidas saludables; b) de AF (Play Smart), diseñado para promover AF en la vida diaria; c) de nutrición y AF (Eat Smart and Play Smart), en el que se trataron temas de nutrición e implemento un programa de AF, y d) de control. Los resultados evidenciaron un mejor conocimiento nutricional en todos los niños, particularmente en los grupos Eat Smart y Eat Smart Play Smart. Se observó también un incremento en la AF durante el descanso de la mañana, siendo más alto en los grupos de intervención incluyendo el de nutrición, comparados con el grupo control. No se evidenciaron diferencias de género en cuanto a la cantidad de actividad. De la misma manera, hubo un aumento en el consumo de frutas y vegetales en los estudiantes (Brug, Oenema y Ferreira, 2005).

El programa Super Kids - Super Fit (Virgilio y Berenson, 1998) fue una intervención basada en los principios de la teoría de aprendizaje de Bandura, con el objetivo de desarrollar comportamientos positivos frente a la realización de la AF. Se diseñó, implementó y evaluó un programa de promoción de salud cardiaca con niños y niñas de escuelas primarias. La intervención incluyó revisión clínica, cambios en el programa del almuerzo escolar, currículo académico de salud cardiovascular, modalidades escolares y comunitarias de intervención, educación familiar, evaluación física (antropometría), antecedentes de factores de riesgo cardiovascular, presión arterial, cuestionarios y exámenes de laboratorio (lipoproteínas, colesterol, hemoglobina) y un programa de condición física cardiovascular denominado superniños - super en forma coordinado por los departamentos de EF de las escuelas participantes, que enfatizaba sobre la AF vigorosa a través de los programas de EF tradicional. En el programa de ejercicio, se realizaban tres veces por semana en sesiones de 30 a 35 minutos. Además, se implementaron estrategias de intervención escolar y comunitaria para generar en los estudiantes la adopción de la AF en el tiempo libre. Las actividades que 
se adelantaron fueron: carreras de observación, día de campo, feria saludable, pronóstico de condición física y trabajo extraclase. Las actividades con los padres estaban encaminadas a promover el conocimiento de estilos de vida saludable, desarrollo de habilidades prácticas, apoyo hacia los niños y la mejora dentro de la familia de los comportamientos positivos frente a la AF.

El Instituto Nacional de Salud (National Institutes of Health) en Carolina del Sur, diseño el programa Lifestyle Education for Activity Program (Leap), basado en el modelo ecológico social derivado de la teoría cognitiva social (Felton et al., 2005) que incluía ambiente escolar, vínculos escuela/comunidad y cambio organizacional. Se desarrollaron clases de EF que incluían actividades de refuerzo de las habilidades comportamentales para la AF, educación en salud. En el ambiente escolar se implementó una cultura de promoción de la AF por parte de docentes y personal de la escuela. A nivel comunitario se convocó a la participación de la familia y de la comunidad en general. Los resultados del estudio indican que el programa cumplió con el objetivo de incrementar la AF en las niñas. El estudio concluye que la intervención tuvo éxito por cuatro razones: a) los enfoques y cambios de contenido de las clases de EF y los temas de educación en salud eran fáciles de desarrollar e implementar; b) participaron todos los estamentos escolares y grupos para cambiar el ambiente y promover la AF en las niñas; c) los cambios a nivel de la escuela eran de bajo costo; y d) los cambios de políticas de la escuela, como permitir que los profesores usaran el tiempo de planificación para realizar actividades físicas, demostró el compromiso de la escuela hacia el cambio.

El proyecto de Salud Escolar de Australia Occidental (WASH, Western Australian School Health Project, 1992-1995) referido por McBride y Midford (1999) se basó en el modelo de Kolbe para promoción en salud en el contexto escolar. Uno de los ejes fundamentales del programa era establecer un comité de salud en cada una de las escuelas participantes, compuesto por profesores, administrativos, padres, estudiantes y otros miembros de la comunidad escolar como el encargado de la cafetería y la enfermera. El proyecto constaba de los siguientes componentes: a) educación escolar en salud; b) EF: currículo, deporte y AF regular; c) ambiente escolar de salud; d) servicio de nutrición y alimentos escolares; e) actividades de promoción en salud para el personal de la escuela; f) actividades integradas entre la escuela y la comunidad para promoción en salud; g) participación de los padres.

La evaluación del impacto se realizó con escuelas involucradas en los últimos tres años del proyecto (1993-1995) y continuó por un periodo de 12 meses, subsecuentes a su conclusión. La evaluación comprendió dos áreas: en la primera se identificaron cambios en el conocimiento, actitud y comportamiento de los participantes en el proyecto: en la segunda, objeto de éste estudio, se observaron los cambios organizacionales a nivel de la escuela y el compromiso de la comunidad (cambios de impacto en la escuela). Según los resultados, la intervención tuvo un impacto inmediato y significativo en varios factores. Por ejemplo, las escuelas de intervención presentaban mayores posibilidades de tener un comité de salud (39 $\%)$, una política actual de salud (26\%), tener personal de tiempo completo para las actividades de promoción en salud ( $8 \%$ ), interactuar con la comunidad local para complementar la actividad de promoción en salud (33 \%) y realizar actividades de promoción en salud para el personal (12\%) que las escuelas del grupo control. Estos niveles de cambio se mantuvieron hasta 12 meses después de la intervención.

El proyecto de Healthy Youth (Dzewaltowski, Esabrooks y Johnston, 2002) se implementó en escuelas de bachillerato para dar un sistema operativo al modelo cognitivo social, a través de intervenciones en salud publica efectivas, basado en el sistema de difusión en el lugar para llegar a los lugares donde los adolescentes pasan la mayor parte de su tiempo en la escuela, y fuera de ella, con el objetivo de identificar los factores que día a día influencian el desarrollo de los ambientes físicos y sociales de los adolescentes. Los pasos de la planeación fueron: elegir un lugar, establecer y documentar un objetivo que responda a una de las siguientes preguntas: ¿Cómo desarrollar un lugar saludable? ¿Cómo contactar y atraer a los participantes a este lugar? A los profesores se les planteó como objetivo implementar temas sobre importancia del consumo de frutas verduras y de AF.

En cuanto a los comportamientos relacionados con AF de niños y adolescentes, existe una serie factores determinantes que proporciona recomendaciones para la construcción de modelos de predicción e intervención (Kohl y Hobbs, 1998). Se hace necesario identificar el potencial de los factores fisiológicos y biológicos como 
determinantes del comportamiento en AF de los niños y adolescentes, para así realizar el diseño de intervenciones apropiadas que puedan ser implementadas y desarrolladas en esta población. Respecto a los factores ambientales, las influencias geográficas y las estaciones, al parecer, desempeñan un papel importante en los comportamientos para la AF. En cuanto a los factores psicológicos, sociales y demográficos, el estudio indica que la autoeficacia ha sido utilizada no solo para correlacionar el comportamiento en AF, sino también para predecir la participación de los adolescentes de los colegios. Además, reporta que los niños que son hijos de padres físicamente activos son más activos que los hijos de padres inactivos y que la influencia de los compañeros es más evidente en niños que en niñas, lo cual puede explicar el rápido descenso de la participación de las niñas en AF durante la adolescencia.

\section{DISCUSIÓN}

La escuela es el escenario donde discurre la vida, la formación, la construcción de hábitos, costumbres y comportamientos de los seres humanos desde los 5 hasta los 18 años. Esto la ubica como un espacio privilegiado para promover la salud y los comportamientos saludables, permitiendo proyectar sociedades adultas más sanas y con mejor calidad de vida. Esta reflexión ha conducido a lo largo de la última década, a la comunidad científica encargada de la promoción de la salud, a volcar sus esfuerzos para diseñar programas de $\mathrm{AF}$ en las escuelas, como una estrategia para fomentar la salud y mantener estilos de vida saludables desde la infancia. Según los resultados referidos en los estudios, se puede establecer que los niños, niñas y adolescentes manifiestan un cambio positivo en sus hábitos cuando se les educa acerca de la importancia de la AF y respecto a cómo mantenerse saludables. Sin embargo, a pesar de los logros reportados en los diversos estudios revisados surgen varios interrogantes que son necesarios discutir.

Los diseños utilizados en las intervenciones descritas utilizan asignación aleatoria y grupo control. La mayoría de los estudios identificados se adelantaron en Estados Unidos, seguidos del Reino Unido, Canadá, Australia y Golfo Pérsico. Así, la mayor parte de las intervenciones se realizaron en los grados más bajos y a medida que aumentaba el grado disminuía el número de estudios, estos resultados concuerdan con la revisión realizada por Stone, Mckenzie, Welk y Booth (1998). Por otra parte, el tiempo de ejecución del programa, la frecuencia y la duración de las sesiones fueron muy heterogéneos.

En 25 propuestas los principales actores fueron los estudiantes, profesores y el grupo de investigadores, únicamente en cuatro estudios se involucró la comunidad escolar, la familia, agentes sociales del entorno escolar y las instituciones del Estado, actores fundamentales para el éxito de estos proyectos.

Las teorías y modelos han sido utilizadas para indagar sobre los factores determinantes en el cambio de comportamiento. Sin embargo, cada una de ellas abordaba esta problemática desde diversas perspectivas: dimensión individual (teorías del aprendizaje, modelo transteórico, estados de cambio y el modelo prevención olvidos y descuido), dimensión interpersonal (teoría de la acción razonada, teoría del comportamiento planeado, teoría del aprendizaje social, y la teoría cognitiva social) y dimensión colectiva (modelos ecológicos). Esta variación en la forma de abordaje del problema parte de la forma de interpretar como se entiende la salud y la promoción de la salud en relación con la AF, por tanto la eficacia de cada uno de ellos es motivo de grandes controversias que no serán abordadas en el presente estudio. Los modelos teóricos utilizados para el diseño de programas de AF para la salud en el contexto escolar presentaron resultados positivos independientemente del modelo utilizado, lo cual dejaría de lado la discusión que a nivel teórico se viene dando sobre la efectividad de un modelo sobre los otros.

En el rastreo bibliográfico se observó que en diferentes estudios se desarrollaron intervenciones sin hacer uso, para su diseño, de modelos teóricos presentando igualmente resultados positivos; esto nos ubica en una problemática compleja y en posturas contradictorias frente al uso de los modelos teóricos para el diseño de programas de promoción de la AF. No se pueden sacar conclusiones frente a la eficacia de unos y otros debido a que las condiciones (duración, frecuencia, agentes involucrados, grado escolar) en que se implementaron los estudios son muy diversas.

Otro elemento en el análisis de los programas fueron las variables que se determinaron para verificar la efectividad de las intervenciones. Los estudios centraron su atención en identificar el incremento en los niveles de AF, disminución del índice de masa corporal 
e incremento en los conocimientos sobre funciones, cardiovasculares, nutrición y riesgos ocasionados por el sedentarismo.

Frente al impacto a largo plazo, únicamente el estudio de Dale y Corbin (2000) evalúa el efecto del programa varios años después, cuando los jóvenes se encuentran en la universidad. Esta reducida evidencia sobre el impacto a largo plazo, convoca la necesidad de realizar estudios longitudinales que conduzcan a dilucidar el verdadero efecto que tiene este tipo de programas en la construcción de hábitos de vida saludables en niños y adolescentes que sean perdurables hasta la edad adulta; además daría elementos explicativos y relaciones sobre las diferentes barreras, motivaciones y factores que conducen al sedentarismo.

El incremento en la AF se registró principalmente en el espacio escolar, evento que puede convertirlo en un elemento distractor, esencialmente en los grupos de niños y niñas. El niño por naturaleza es un ser en movimiento, que requiere de este para explorar el mundo, para conocerlo, para construirse y para establecer relaciones con sus pares. La escuela emerge como escenario regulador de sus actividades motrices limitándolas en el tiempo y espacio, al disponer los niños de mayor tiempo para realizarlas, debido a la implementación de estos proyectos, acarrea como consecuencia lógica un mayor nivel de AF, lo cual conduce a plantearse la pregunta: ¿Este mayor nivel de AF es debido a las bondades del programa, o es simplemente una respuesta natural del niño a su necesidad de movimiento? ¿Estos programas mediatos o puntuales logran estructurar hábitos saludables?

Con este propósito, Johnson y Deshpande (2000) plantean que la influencia de la educación recibida en la escuela es determinante en la adquisición o modificación de hábitos alimenticios, higiénicos y de AF, que se afianza a lo largo del proceso educativo perdurando estas conductas en la adultez. Sin embargo, la construcción de hábitos se fundamenta en un proceso educativo, mediado por aspectos individuales, interpersonales, organizacionales, comunitarios, urbanísticos y políticos, lo cual permite identificar los mediadores y las estrategias de cambio y dar una nueva mirada a los factores regulados por la cultura (visión antropológica) como los mitos, imaginarios, prácticas, hábitos y costumbres frente a la AF.
Algo importante en el conjunto de estudios es el valor que adquiere la clase de EF como el epicentro para el desarrollo e implementación de los programas. Esta perspectiva resalta la importancia que tiene el docente de EF en el cuidado y promoción de la salud. Sin embargo, esta reflexión no surge de la EF, el conjunto de estudios revisados indica que la creación y diseño de estos programas surgió de hospitales y facultades de salud. La Organización Mundial de la Salud (OMS), en su carta de promoción de la salud, ubicó a la escuela como un elemento fundamental para la promoción de la salud: “[...] los centros educativos intervendrán fundamentalmente en el desarrollo de las habilidades personales, la creación de entornos favorables a la salud y el fortalecimiento de la acción comunitaria" (oMs, 1986, s.p.) Posteriormente, en 1996 planteó la iniciativa de escuelas promotoras de salud que fortaleció la promoción y educación en salud en todos los niveles educativos y en los espacios donde la comunidad escolar aprende, trabaja, juega y convive, estimulando la cooperación entre el sector educativo y el de salud. En 2002 en la V Conferencia Europea de la Unión Internacional de Promoción de la Salud y Educación en Salud (UIPES) celebrada en Londres, se reafirmaron y fortalecieron los postulados anteriores.

El documento Healthy People 2010, editado por el USA Departement of Health and Human Services (2000), indica que la importancia de la EF en la promoción de la salud está sustentada en tres objetivos: aumentar la proporción de escuelas públicas y privadas con EF diaria para todos los estudiantes, aumentar la cantidad de niños y adolescentes que participen en sesiones diarias de EF escolar e incrementar el porcentaje de niños y adolescentes que estén activos, al menos el $50 \%$ de la duración de la clase de EF. En Colombia el sector educativo, en el documento de trabajo Lineamientos para la educación en estilos de vida saludables, publicado en 1997, desarrolla las formas y bases para la inclusión de la temática de estilos de vida dentro del Proyecto Educativo Institucional. Se sugieren algunas actividades en coordinación con otros sectores para que estudiantes, docentes y comunidad contribuyan a la construcción de una cultura de la salud y faciliten desde temprana edad, la formación de estilos de vida saludables.

Estos lineamientos convierten la salud en objetivo de la escuela, que requiere abordarse de forma transversal, por tanto debe estar presente en el currículo escolar. 
Con este propósito, la Aiesep World Convention, realizada en 1990, planteó que en el currículo escolar la EF cumple un papel importante en la promoción de la salud. Esto exige reflexionar sobre los objetivos, contenidos, actividades, metodología y evaluación de la asignatura, para adecuarlos a la noción de salud. Estos elementos, propuestos por Cantera y Devis (2002), se evidencian en los diferentes estudios revisados en los cuales una de las actividades de los programas era implementar dentro de la clase de EF conocimientos sobre fisiología del corazón, anatomía, nutrición AF y salud.

Esta perspectiva no es nueva, históricamente la EF ha tenido un rol importante en la educación en salud en Estados Unidos. En el primer número de la revista Common School Journal, publicado en 1838, se planteó que la EF aportaba el conocimiento para comprender las leyes y las funciones del cuerpo sano; diez años después (1850) surgió el informe Shattuck, hito importante de la salud pública, que recomendaba que a todos los niños se les debía enseñar tempranamente la forma de preservar la salud (Tappe y Burgeson, 2004). Esta demanda social de promoción de la salud a través de la EF escolar en la sociedad contemporánea continúa presente (SimonsMorton, O’hara, Simons-Morton y Parcel,1987; Sallis y McKenzie, 1991; Baranowski, Bouchard, Bar-Or, Bricker y Heath, 1992; Walsh y Tilford, 1998; Cale, 2000; Johnson y Deshpande, 2000; Bulger, Mohr, Carson y Wiegand, 2001; Wallhead y Buckworth, 2004; Cale y Harris, 2006; Lee, Burgeson, Fulton y Spain, 2007; Murillo et al., 2013).

A pesar de estas evidencias, la promoción de la salud requiere la reformulación de los paradigmas que organizan el campo de la salud y la EF en el nuevo contexto sociocultural, lo cual implica para los profesores de EF, una integración de la promoción de la salud en el campo de la EF. Esto conduce a preguntarse si los profesores de EF disponen de una formación adecuada, para asumir este reto. Las investigaciones realizadas por Anderson y Thorsen (1998); Walsh y Tilford (1998); Bulger et al. (2001); Fontana y Apostolidou (2001); Burgeson et al. (2003) indican una deficiente formación de los profesores de EF como educadores en salud y coinciden que los programas de formación del profesorado no son adecuados para afrontar esta responsabilidad profesional.

\section{CONCLUSIONES}

El análisis de la literatura frente a los programas de actividad física para la salud en el contexto escolar conduce a realizar una propuesta de modelo de intervención. Esta se estructura a partir del modelo ecológico adaptado por el Celafics en Brasil. Tres componentes centrales orientan la construcción del modelo alrededor de los cuales se construirán las actividades y estrategias: el intrapersonal, el medio ambiente social y el medio ambiente físico.

\section{Componente intrapersonal}

Este componente está orientado a generar y consolidar cambios en los comportamientos y hábitos de los niños frente a la AF, a partir del desarrollo de actitud positiva, autoeficacia y autonomía. En el desarrollo de la actitud positiva se pretende que tanto niños como adolescentes comprendan y conozcan la importancia de la AF para la salud y las consecuencias del sedentarismo. Se sugiere desarrollar contenidos teóricos en la clase de EF, sobre la importancia de la AF para la salud, factores de riesgo y sedentarismo, anatomía y funcionamiento del sistema cardiovascular. En términos de difusión se pueden utilizar varias estrategias como: programas en la emisora escolar, murales, creación de una página en internet, redes sociales, etc. El desarrollo de la autoeficacia busca el inicio de la construcción de hábitos de vida activos, es decir que los escolares desarrollen o incrementen el gusto por las actividades físicas, que pude ser a través de la implementación de nuevos deportes, o prácticas corporales alternativas, con una periodicidad de cinco días a la semana. La autonomía está encaminada a incrementar los niveles de actividad física extraescolar, mejorar las cualidades físicas y un uso activo del tiempo libre.

\section{Componente del ambiente social}

Este componente está orientado a generar una cultura institucional de actividad física para la salud generando ambientes y entornos que conduzcan a promover cambios de comportamiento. Para ello se deben generar políticas y recursos desde tres contextos ambientales: microsistema, mesosistema y ecosistema. El microsistema comprende las acciones que se deben llevar a cabo en la clase de educación física y el descanso. Estas deben estar constituidas por un programa de actividad física para la salud que conduzca a incrementar el nivel de 
actividad y participación en la clase de educación física, y un programa de formación para los docentes en temas relacionados con actividad física y salud, estrategias para la construcción de hábitos saludables, nuevos deportes y modalidades de actividad física.

El mesosistema involucra el conjunto de acciones que se deben llevar a cabo desde la escuela hacia la familia, como talleres de sensibilización e información sobre actividad física y salud, programas de actividad física para padres e hijos (caminatas ecológicas, deportes, danzas, etc.).

El ecosistema involucra las políticas y estrategias que las directivas del colegio debe asumir para la generación de entornos y ambientes saludables; por ejemplo: programa de actividades extraescolares; incentivos para los estudiantes, docentes y funcionarios más activos de la institución; asignación de horas para los docentes de la institución que diseñen e implementen el programa; talleres a directivos y funcionarios del colegio sobre actividad física y salud; programa de actividad física y salud para directivos y funcionarios; asignación de espacios y recursos; destinación de una hora diaria para la clase de EF.

\section{Componente ambiente físico}

Este componente tiene como objetivo realizar un análisis del medio ambiente natural, como el clima y la geografía; medio ambiente construido, infraestructura y escenarios deportivos de los institutos públicos del deporte a los cuales se pueda acceder.

\section{REFERENCIAS BIBLIOGRÁFICAS}

Anderson, A. y Thorsen, E. (1998). Pre-service teacher education in health education: An Ontario Survey. Journal of Education for Teaching: International Research and Pedagogy 24(1), 73-74.

Agron, P.; Takada, E. y Purcell, A. (2002). California project LEAN's food on the run program: An evaluation of a high school-based student advocacy nutrition and physical activity program. J Am Diet Assoc 102(3), 103105.

Baranowski, T.; Bouchard, C.; Bar-Or, O.; Bricker, T. y Heath, G. (1992). Assement, prevalence and cardiovascular benefits of physical activity and fitness in youth. Medicine and Science in Sport and Exercice 24(supl), 237-247.

\section{Evaluación y seguimiento}

Para verificar el impacto del programa es necesario generar un sistema de evaluación que dé cuenta del impacto en los tres componentes, en la generación de hábitos saludables, en el incremento de la cantidad de AF, desarrollo de las cualidades físicas (resistencia cardiovascular, fuerza y flexibilidad). Este proceso se debe realizar al comienzo del programa, y cada semestre acorde al calendario de la institución educativa. Esto permitirá analizar su comportamiento en cada fase y retroalimentar el programa con los respectivos ajustes. Igualmente se debe contemplar un seguimiento del impacto del programa a largo plazo, que permitan verificar el nivel de actividad física que tienen los niños y niñas años después de la participación en el programa.

Aspectos generales del programa

La elaboración del diseño general debe contar con la participación de profesores estudiantes, padres de familia y directivas de la institución. Este diseño debe generar las políticas estrategias y recursos con que contará el programa. Posteriormente cada curso con la participación de padres de familia, profesores y estudiantes diseñará las actividades a realizar.

Bayne, M.; Fardy, P.; Azzollini, A.; Magel, J.; Schmitz, K. y Agin, D. (2004). Improvements in hearth health behaviors and reduction in coronary artery disease risk factors in urban teenaged girls through a schoolbased intervention: The PATH program. Am J Public Health 94(9), 1538-1543.

Beets, M. y Pitetti, K. (2002). Contribution of physical education and sport to health-related fitness in high school students. J Sch Health 75(1), 25-30.

Brug, J.; Oenema, A. y Ferreira, I. (2005). Theory, evidence and intervention mapping to improve behavior nutrition and physical activity interventions. Int $J$ Behav Nutr Phys Act. 2(2), 1-7. 
Bulger, S.M.; Mohr D.J.; Carson, L.M. y Wiegand, R.L. (2001). Insufising health -related physical fitness in physical education teacher education. Quest 53(4), 403-417.

Burgeson, C.R.; Wechsler, H.; Brener, N.D.; Young, J.C. y Spain, C.G. (2003). Physical Education and Activity: Results from the school health policies and programs Study 2000. Journal of Physical Education, Recreation and Dance 74(1), 20-36.

Cale, L. (2000). Physical activity promotion in secondary schools. European Physical Education Review 6(1), 71-90.

Cale, L. y Harris, J. (2006). School-based physical activity interventions: effectiveness, trends, issues, implications and recommendations for practice. Sport, Education and Society 11(4), 401-420.

Cantera, M.A. y Devis-Devis, J. (2002). La promoción de la actividad física relacionada con la salud en el ámbito escolar. Implicaciones y propuestas a partir de un estudio realizado entre adolescentes. Apuntes de Educación Física y Deportes, 67, 54-62.

Cardon, G.M. y DeBourdeaudhuij, I.M. (2002). Physical educaction and physical activity in elementary schools in Flanders. European Journal of Physical Education $7(1), 5-18$.

Chad, K.E.; Humbert, M. y Jackson, P. (1999). The effectiveness of the Canadian Quality Daily Physical Education Program on School Physical Education. Res Q Exerc Sport 70(1), 55-64.

Corbin, C.B. y Pangrazi, R.P. (2004). Physical Activity for Children: Current Patterns and Guidelines. Research Digest 5(2), 2-8.

Dale, D. y Corbin, C. (2000). Physical activity participation of high school graduates following exposure to conceptual or traditional physical education. Res $Q$ Exerc Sport 71(1), 61-68.

Devís, J. y Peiró, C. (1992). Exercise and health in a Spanish PE Curriculum: a modified programme of 'the exercise challenge'. En: T. Williams, L. Almond y A. Sparkes (eds.). Sports and physical activity: moving towards excellence (pp. 418-428). Londres: E y FN Spon.

Datar, A. y Sturm, R. (2004). Physical Education in Elementary School and Body Mass Index: Evidence from the Early Childhood Longitudinal Study. Am J Public Health 94(9), 1501-1506.

Department of Health and Human Services. Department of Education. Centers for Disease Control and Prevention. National Center for Chronic Disease Prevention and Health Promotion. (2000). Promoting Better Health for Young People Through Physical
Activity and Sports. Recuperado en julio de 2009, de: www.cdc.gov/nccdphp/dash/presphysactrpt

Dobbins, M.; Husson, H.; DeCorby, K. y LaRocca, R.L. (2013). School-based physical activity programs for promoting physical activity and fitness in children and adolescents aged 6 to 18. Cochrane Database Syst Rev, 2, Cd007651.

Dowda, M.; Sallis, J.; McKenzie, T.; Rosengard, P. y Col, H. (2005). Evaluating the Sustainability of SPARK Physical Education: A Case Study of Translating Research into Practice. Res Q Exerc Sport 76(1), 11-19.

Dzewaltowski, D.; Esabrooks, P. y Johnston, J. (2002). Healthy youth places promoting nutrition and physical activity. Health Educ Res. 17(5), 541-551.

Felton, G.; Saunders, R.; Ward, D. y Dishman, R. (2005). Promoting physical activity in girls: A case study of one Sschool's success. J Sch Health 75(2), 57-62.

Fernández, J.A.; González, M. y Farfán, M. (2011). Modelo teórico, agentes, estrategias, duración, escenarios para la implementación, grado escolar e indicadores de resultados utilizados en los programas de promoción de la actividad física en el contexto escolar. Revista Medunab 14(2), 26-37.

Fernández, J.A. y Hoyos, L.A. (2007 ). Perfil de las Cualidades Fisicas y Antropometricas de los Escolares Colombianos. 1a. ed. Bogota: Universidad Pedagógica Nacional.

Fernandez, J.A. y Rocha, A. (2008). Teorías y modelos utilizados para el diseño de programas de actividad física para la salud. En: H. Cardenas (ed.). Visiones sobre medicina comunitaria (pp. 241,265). Bogota: Editorial Universidad del Bosque.

Fernandez, J.A. y Ruiz, F.A. (2012). Estudio transversal de crecimiento de los escolares bogotanos: Valores de estatura, peso e índice de masa corporal de los siete a los dieciocho años. Universidad y Salud 1(15), 21-41.

Fontana, D. y Apostolidou, M. (2001). Perspectives of serving teachers on the respective importance of areas deemed suitable for inclusion in the health education curriculum for Cyprus schools. Health Education Journal 60(2), 173-183.

Fowler-Brown, A. y Kahwati, L. (2004). Prevention and treatment of overweight in children and adolescents. Am Fam Physician 69(11), 2591-2598.

Grow, H.M.; Saelens, B.E.; Kerr, J.; Durant, N.H.; Norman, G.J. y Sallis, J.F. (2008). Where are youth Active? Roles of proximity, active transport, and built environment. Medicine y Science in Sports y Exercised, 40(12), 20722079. 
Harrell, J.; Gansky, S.; McMurray, R.; Bangdiwala, S.; Frauman, A. y Bradley, C. (1998). School based interventions improve heart health in children with multiple cardiovascular disease risk factors. Pediatrics 102(2), 371-380.

Harrell, J.S.; McMurray, R.; Gansky, S. y Bangdiwala, S. (1999). A public health vs a risk-based intervention to improve cardiovascular health in elementary school children, the cardiovascular health in children study. Am J Public Health, 89(10), 1529-1535.

Higgings, J.; Gaul, C.; Gibbons, S. y Gyn, G. (2003). Factors Influencing Physical Activity Levels Among Canadian Youth. Can J Public Health 94(1), 45-51.

Hill, G. y Quam, B. (2003). A Comparison of current and Ideal Fitness Promotion Strategies in Washington State. Journal of Physical Education, Recreation y Dance 74(8), 39-54.

Hutchinson, G.; Freedson, P.; Ward, A. y Rippe, J. (1990). Ideal to real. Implementing a youth fitness program. Journal of Physical Education, Recreation y Dance 61(6), 52-58.

Johnson, J. y Deshpande, C. (2000) Health education and physical education: Disciplines preparing stundents as productiva, healthy citizens for the challenges of the 21st century. Journal of School Health 70(2), 66-68.

Kohl, H.W. y Hobbs, K. (1998). Development of Physical Activity Behaviors Among Children and Adolescents. Pediatrics 101(3), 549-554.

Lee, S.M.; Burgeson, C.R.; Fulton, J.E. y Spain, C.G. (2007). Physical Education and Physical Activity: Results From the School Health Policies and Programs Study 2006. Journal of School Health 77(8), 435-463.

Leslie, J.; Yancy, A.; McCarthy, W. y Albert, S. (1999). Development and implementation of a school-based nutrition and fitness promotion program for ethnically diverse middle-school girls. Am J Public Health 99(8), 967-970.

Levin, S. y Martín, M. (2002). Catch the Catawba SPARK: Physical activity for head start youths. Journal of Physical Education, Recreation y Dance 73(3), 39-42.

Levin, S.; Martin, M.; McKenzie, T. y DeLouise, A. (2002). Assessment of a pilot video's effect on physical activity and heart health for young children. Family and community health. Fam Community Health, 25(3), 10-17.

Marston, R. (2002). Addressing the university's tripartite mision through an early childhood movement program. Journal of Physical Education, Recreation y Dance 73(5), 35-41.

McBride, N. y Midford, R. (1999). Encouraging schools to promote health: Impact of the western Australian school health project (1992-1995). J Sch Health 69(6), 220-226.
McBride, N.; Midford, R. y Cameron, I. (1999). An empirical model for school health promotion: the Western Australian school health project model. Health Promot Int, 14(1), 17-25.

McKenzie, T.; Feldman, H.; Woods, S. y Romero, K. (1995). Children's activity levels and lesson context during third-grade physical education. Res Q Exerc Sport. 66(3), 184-194.

McKenzie, L.; Sallis, J.; Elder, J.; Berry, C.; Hoy, P.; Nader, P. y Broyles, S. (1997). Physical activity levels and prompts in young children at recess: A two-year study of a biethnic sample. Res Q Exerc Sport. 68(3), 195-202.

Mendoza, R.; Ságrera, M. y Batista, J.M. (1994). Conductas de los escolares españoles relacionadas con la salud (19861990). Madrid: Consejo Superior de Investigaciones Cientificas.

Mummery, W.; Spence, J. y Hudec, J. (2000). Understanding physical activity intention in canadian school children and youth: Application of the theory of planned Behavior. Res Q Exerc Sport. 71(2), 116-124.

Murillo, B.; García, E.; Generelo, E.; Bush, P.L.; Zaragoza, J.; Clemente, J.A. y García L. (2013). Promising school-based strategies and intervention guidelines to increase physical activity of adolescents. Health Education Research 28(3), 523-538.

Nabipour, I.; Miami, S.; Mohammadi, M.; Heidari, G. y Bahramian, F. (2002). A school-based intervention to teach 3-4 grades children about healthy heart, The Persian gulf healthy heart project. Indian J Med Sci 72(5), 199-204.

National Center for Chronic Disease. (2002). Prevention and Health Promotion, Nutrition and physical activity. Kids-walk-to-school. In National Center for Chronic Disease

Pangrazi, R.; Beighle, A.; Beige, T. y Vack, C. (2003). Impact of promoting lifestyle activity for youth (PLAY) on children's physical activity. J Sch Health 73(8), 317321.

Prieto, A. (2003). Modelo de Prevención de la Salud, con Énfasis en Actividad Física, para una comunidad estudiantil universitaria. Rev. Salud Pública 5(3), 284300.

Reinhardt, W. y Brevard, P. (2002). Integrating the food guide pyramid and physical activity pyramid for positive dietary and physical activity behaviors in adolescents. J Am Diet Assoc 102(3), S96-S99.

Sallis, J. y McKenzie, T. (1991). Physical education'srole in public health. Research Quarterly for Exercise and Sport 62(2), 124-137.

Sallis, J.; McKenzie, T.; Alcaraz, J.; Kolidy, B. y Faucette, N. (1997). The effects of a 2-year physical education 
program (SPARK) on physical activity and fitness in elementary school students. Am J Public Health 87(8), 1328-1334.

Simons-Morton, B.G.; O'hara, NM.; Simons-Morton, D.G. y Parcel, G.S. (1987). Children and fitness: a public health perspective. ResearchQuarterly for Exercise and Sport, 58, 295-302.

Simons-Morton, B.G.; Parcel, G.S.; Baranowski, T.; Forthofer, R. y O'hara N. (1991). Promoting physical activity and healthful diet among children: results of a school-based intervention study.American Journal of Public Health 81(8), 986-991.

Skybo, T.A. y Ryan N. (2002). A School-based intervention to teach third grade children about the prevention of heart disease. J Pediatr Nurs. 28(3), 223-235.

Staunton, C.E.; Hubsmith, D. y Kallins, W. (2003). Promoting safe walking and biking to school: The Marin county success story. Am J Public Health 93(9), 1431-1434.

Stewart, J.; Dennison, D.; Kohl, H. y Doyle, J. (2004). Exercise level and energy expenditure in the take $10 \AA$ in-class physical activity program. J Sch Health 74(10), 397-400.

Stone, E.J.; McKenzie, T.L.; Welk, G.J. y Booth, M.L. (1998). Effects of physical activity interventions in youth. Review and synthesis. Am J Prev Med 15(4), 298-315.
Tappe, M.K. y Burgeson, C.R. (2004). Physical education: A cornerstone for physically active lifestyles. Journal of Teaching in Physical Education, 23, 281-299.

Virgilio, S.J. y Berenson, G. (1998). Super Kids-Superfit. A comprehensive fitness intervention model for elementary schools. Journal of Physical Education, Recreation y Dance 59(8), 19-25.

Wallhead, T.L. y Buckworth, J. (2004). The role of physical education in the promotion of youth physical activity. QUEST, 56, 285-301.

Walsh, S. y Tilford, S. (1998). Health education in initial teacher training at secondary phase. In England and Wales: current provision and the impact of the 1992 government reforms. Health Education Journal 57(4), 360-373.

Warren, J.; Lightowler, S.; Bradshaw, M. y Perwaiz, S. (2003). Evaluation of a pilot programme aimed at the prevention of obesity in children. Health Promot. Int. 18(4), 287-296.

World Organization Health (WHO) (1986). Ottawa Charter for Health Promotion. Ottawa: wHo, Health and Welfare Canada. Canadian Public Health Association.

Wu, T. y Jwo, J. (2005). A prospective study on changes of cognitions, interpersonal influences, and physical activity in Taiwanese youth. Res $Q$ Exerc Sport. 76(1), $1-10$. 